\title{
0642 BATHING BURN INJURIES IN CHILDREN: AN OPPORTUNITY FOR TEACHING AND PREVENTION
}

R Gonzalez*, K B Nagappala, H Schaewe, C M Shanti Correspondence: Department of Paediatric Surgery, Children's Hospital of Michigan, Carl's Building, 3902 Beaubien Street, Detroit, MI 48201, USA

10.1136/ip.2010.029215.642

Introduction Bath-related burn injuries are a significant cause of morbidity in children. Despite the common notion that sinks are smaller, and hence harmless, both bathtubs and sinks remain culprits. We sought to review the experience at our paediatric burn centre in an effort to analyse the mechanisms of burn injury while bathing and enhance our current education strategies.

Methods A 5 year retrospective analysis of the burn registry at our institution was conducted from 2004 to 2008. A thorough analysis of patient demographics, burn related data (burn depth, distribution and percent of total body surface area (TBSA)), operative intervention and child protective services (CPS) involvement was performed. Statistical analysis was conducted using SPSS.

Results During this time period, 1237 patients incurred burn injuries. The incidence of bath associated burns was $8 \%(n=104)$. Seventy two patients $(69 \%)$ were injured while in the bathtub, 31\% were burned in the sink. The average age of our patients was 15 months, no gender difference was observed. TBSA ranged from $6.5 \%$ to $10.3 \%$. Greater than $90 \%$ suffered partial thickness burns; greater than $50 \%$ favoured the lower body, regardless of the mechanism. No association between mechanism of injury and depth of burn was observed $(p=0.105)$. CPS involvement was sought in more than half of the cases, irrespective of the mechanism of injury. Although length of stay (LOS) was similar with both types of injuries, it was associated to CPS involvement $(p=0.015)$ and the need for operative intervention $(p=0.000)$. (table 1)

Conclusions Our series confirms the morbidity associated to bath-related burn injuries in the paediatric population. Both bathtubs and sinks pose similar risks in terms of burn depth, distribution, body surface area involvement and the need for surgical intervention. It is our responsibility as healthcare providers to continue enhancing care provider education for safe measures while bathing children via in hospital teaching as well as prevention outreach programs. Table 1 patient demographics of bathtub versus. sink burns Variable Mechanism of injury p value bathtub sink 72 (69\%) 32 (31\%) gender (male) 40 (56\%) 17 (53\%) 0.82 age (years) 1.582.51 1.351.28 0.63 burn distribution 38 (53\%) 19 (59\%) 0.75 (lower body) burn depth 65 (90\%) 30 (94\%) 0.26 (partial thickness) TBSA $10.2713 .41 \%$ 6.4710 .810 .12 operative intervention 10 (14\%) 4 (3\%) 0.85 CPS involvement 39 (54\%) 19 (59\%) 0.83 LOS (days) 8.2611 .48 9.3814.04 0.67. 